\title{
THE MEDICAL PERSPECTIVE ON BURNOUT
}

\section{TAMAR KAKIASHVILI' ${ }^{1}$ JERZY LESZEK ${ }^{2}$, and KRZYSZTOF RUTKOWSKI ${ }^{3}$}

\author{
${ }^{1}$ Sudbury Therapy, Psychotherapy, Ontario, Canada \\ ${ }^{2}$ Wroclaw Medical University, Wrocław, Poland \\ Department of Psychiatry \\ ${ }^{3}$ Jagiellonian University Medical College, Kraków, Poland \\ Department of Psychotherapy
}

\begin{abstract}
Objective: The aim of this study was to review recent medical findings related to burnout, its diagnosis, treatment, characteristic pathophysiological features, and preventive measures. Materials and Methods: A systematic review of the scientific literature in PubMed/Medline was performed. The most recent and important findings were reported. Results: Burnout was found to be a risk factor for myocardial infarction and coronary heart disease. It was also related to reduced fibrinolytic capacity, decreased capacity to cope with stress and hypothalamic-pituitary-adrenal (HPA) axis hypoactivity. Severe burnout symptoms are associated with a lower level or smaller increase of the cortisol awakening response (CAR), higher dehydroepiandrosterone-sulphate (DHEAS) levels, lower cortisol/DHEAS ratios and stronger suppression as measured by the dexamethasone suppression test (DST). More and more literature works suggest that the evaluation of the HPA axis should be brought to the attention of primary care physicians. There is no universal agreement on specific treatment and diagnostic measures to evaluate the wide range of HPA axis disorders. The cost-effective evaluation of adrenal hormones via saliva samples by a primary care physician may significantly alter the course of therapy in numerous chronic disease patients. Psychiatric disorders may have similar symptoms, but they have distinctive hormonal profiles. Having burnout recognized as a medical condition would help in differentiating burnout from similar clinical syndromes, such as depression or anxiety, and provide appropriate treatment to burnout patients. Proper treatment is essential for a fast and full recovery. Conclusion: Chronic stress-related disorders often fall outside the category of a "true" disease and are often treated as depression or not treated at all. The evaluation of adrenal hormones via saliva samples helps to predict burnout. Burnout screening techniques, dietary and nutritional guidelines and lifestyle changes for supporting the HPA function need to be developed. The presented material includes hormonal, dietary, and pharmaceutical perspectives.
\end{abstract}

Key words:

Burnout, Stress, Pathophysiology, Cortisol

\section{SUMMARY}

Burnout can cause serious health implications; the current situation in burnout prevention and treatment could be improved.

Burnout and depression have similar symptoms; burnout is often treated as depression or not treated at all.

The saliva cortisol test may help in predicting burnout and differentiating it from depression.

\section{CONSIDERATIONS}

Diagnosing a patient with burnout is problematic since burnout is not recognized as a mental illness and it is not listed in the Diagnostic and Statistical Manual of Mental Disorders (DSM IV-TR, 2000).

The use of antidepressants in burnout remains controversial. There is no consensus about the diagnostic and treatment measures of burnout.

Received: September 15, 2012. Accepted: December 20, 2012.

Corresponding author: T. Kakiashvili, Sudbury Therapy, Psychotherapy, 885 Regent Street, Sudbury, Ontario, Canada P3E 5M4 (e-mail: info@SudburyTherapy.com). 


\section{INTRODUCTION}

Burnout is specified by the International Classification of Diseases, 10th Revision, Clinical Modification (ICD-10$\mathrm{CM})$ under "Factors influencing health status and contact with health services (Z00-Z99)" as a "State of vital exhaustion" (Z73.0) and identified as a problem related to "life-management difficulty". Vital exhaustion is defined as a state characterized by lack of energy, increased irritability, and demoralization [1]. Maslach [2] stated that burnout is a result of the prolonged response to chronic emotional and interpersonal stressors at the workplace, and it is defined by three dimensions of exhaustion, cynicism, and inefficacy. The ICD-10-CM does not identify "vital exhaustion" as a separate diagnostic entity. A "State of vital exhaustion" is listed under the ICD-10-CM Diagnosis Code Z73.0 (burnout). Therefore, according to the ICD-10-CM, "vital exhaustion" refers to "burnout". However, some researchers prefer using the term "burnout" when it is related to work overload and "vital exhaustion" when it is linked to family and financial stress [3]. Burnout is not a recognized disorder in the Diagnostic and Statistical Manual of Mental Disorders (DSM IV-TR, 2000). The lack of reference to burnout in the DSM IV-TR has important implications.

The presented material includes hormonal, dietary, and pharmaceutical perspectives. It is a call for more research and for developing new treatment approaches. Burnout adversely influences normal functioning. As for modern society illnesses, burnout cannot be traced back to one single cause. It is tied to a variety of risk factors. Feelings of inadequate control over one's work, frustrated hopes and expectations, and low levels of satisfaction seem to be independent contributors to burnout. The burnout syndrome may be seen as a progressively-developed process resulting from the use of relatively ineffective coping strategies with which professionals try to protect themselves from work-related stress.
It has been shown to be a risk factor for myocardial infarction [4] and the coronary heart disease [1]. Vital exhaustion (understood as burnout according to ICD-10-CM) is related to personality type D [5], which is defined as "negative affectivity and the tendency to inhibit the expression of this affect in social interaction".

Vital exhaustion has also been related to reduced fibrinolytic capacity [6], hypothalamic-pituitary-adrenal (HPA) axis hypoactivity and decreased capacity to cope with stress [7]. Vital exhaustion was assessed by the Maastricht Questionnaire [4]. Subjects who scored in the upper third were labeled as exhausted and compared with those who scored in the lower or middle third. Nicolson and van Diest [8] found subtle HPA hypoactivity in vital exhaustion which might have been a result of chronic stress and associated sleep disturbances. Researchers evaluated general cognitive ability, memory and attention in 67 female patients treated for chronic burnout [9]. The patients and 15 healthy control subjects were tested with standardized tests of verbal and nonverbal cognitive ability (WAIS), verbal (Claeson-Dahl) and nonverbal (Rey complex figures) memory, and visual and auditory attention (IVA). Significant reductions in nonverbal memory and auditory and visual attention were noted in the patient group. Researchers suggest that explorations of possible neuroendocrine abnormalities as well as brain imaging may shed further light on the pathophysiology and putative treatment regimes in this disorder.

Esterling et al. [10] examined the long-term physiological consequences of chronic stressors, demonstrating that the physiological and psychological consequences of chronic stressors may persist well beyond the cessation of the actual stressor.

There is no doubt that burnout has serious negative consequences. However, it seems that current medical knowledge and practice are deficient in developing effective treatments for burnout. In this paper, we present the current medical perspective on burnout. 


\section{Recognizing burnout}

There are a number of important decisions to be made when a patient visits a family doctor to seek help. The first decision is how to diagnose the disorder since the patient is rarely aware that he/she suffers from it. On the other hand, it is unrealistic for the family doctor to observe patients at work and, even if it were possible to do so, the chances of diagnosing burnout during a doctor's visit would be next to none since people perform differently when observed at work. They do not seek help until burnout begins influencing their work performance so badly that their employment may be in jeopardy.
It is not uncommon for a family physician to mistake burnout for depression. As we will see, such a mistake may have devastating consequences for the patient. In clinical practice, it is important for a doctor to recognize burnout and distinguish it from disorders which may have similar symptoms (see Table 1).

An interview with a patient may result in considering the administration of a burnout questionnaire. There are a number of psychological scales for burnout available. The most popular is the Maslach Burnout Inventory (MBI) [11]. Using the MBI, it is possible to measure the level of cynicism, exhaustion, and feelings of professional efficacy.

Table 1. Symptoms commonly found in burnout, mood and anxiety disorders

\begin{tabular}{|c|c|c|c|c|c|}
\hline Symptoms & $\begin{array}{c}\text { Major } \\
\text { depressive } \\
\text { disorder }\end{array}$ & $\begin{array}{c}\text { Generalized } \\
\text { anxiety } \\
\text { disorder }\end{array}$ & $\begin{array}{c}\text { Post- } \\
\text { traumatic } \\
\text { stress disorder }\end{array}$ & $\begin{array}{c}\text { Acute stress } \\
\text { disorder }\end{array}$ & Burnout \\
\hline Depressed mood & char & $\mathrm{c}$ & $\mathrm{c}$ & & $\mathrm{s}$ \\
\hline Loss of interest or pleasure & char & & c & & $\mathrm{c}$ \\
\hline Demoralization / subjective incompetence & & & c & & char \\
\hline Anxiety & $\mathrm{c}$ & char & char & char & $\mathrm{c}$ \\
\hline Derealization & & & char & char & $\mathrm{c}$ \\
\hline Depersonalization & & & char & char & $\mathrm{c}$ \\
\hline Changes in appetite or weight & $\mathrm{c}$ & $\mathrm{c}$ & $\mathrm{c}$ & $\mathrm{c}$ & $\mathrm{c}$ \\
\hline Insomnia or hypersomnia & $\mathrm{c}$ & $\mathrm{c}$ & $\mathrm{c}$ & $\mathrm{c}$ & $\mathrm{c}$ \\
\hline Psychomotor agitation or retardation & $\mathrm{c}$ & $\mathrm{m}$ & & $\mathrm{m}$ & $\mathrm{c}$ \\
\hline Fatigue or loss of energy & $\mathrm{c}$ & $\mathrm{c}$ & $\mathrm{c}$ & $\mathrm{c}$ & char \\
\hline Feelings of worthlessness, excessive or inappropriate guilt & $\mathrm{c}$ & & $\mathrm{c}$ & & $\mathrm{c}$ \\
\hline Diminished ability to think or concentrate, or indecisiveness & $\mathrm{c}$ & $\mathrm{c}$ & $\mathrm{c}$ & $\mathrm{c}$ & $\mathrm{c}$ \\
\hline Memory impairment & $\mathrm{c}$ & $\mathrm{c}$ & d & d & $\mathrm{c}$ \\
\hline $\begin{array}{l}\text { Recurrent thoughts of death, suicidal ideation, or suicide } \\
\text { attempt }\end{array}$ & $\mathrm{c}$ & & $\mathrm{c}$ & & $\mathrm{c}$ \\
\hline $\begin{array}{l}\text { Significant impairment in social, occupational, and other } \\
\text { important areas of functioning. }\end{array}$ & $\mathrm{c}$ & $\mathrm{c}$ & c & & $\mathrm{c}$ \\
\hline Somatic complaints & $\mathrm{c}$ & $\mathrm{t}$ & $\mathrm{c}$ & & $\mathrm{c}$ \\
\hline Autonomic hyperarousal and exaggerated startle response & & 1 & $\mathrm{c}$ & $\mathrm{c}$ & $\mathrm{c}$ \\
\hline Increased irritability & $\mathrm{c}$ & $\mathrm{c}$ & $\mathrm{c}$ & & char \\
\hline
\end{tabular}

char - characteristic; c - common; $\mathrm{s}$ - sometimes present; $\mathrm{m}$ - motor restlessness; $\mathrm{d}$ - dissociative amnesia;

$\mathrm{t}$ - muscle tension; 1 - less prominent.

Based on DSM-IV-TR diagnostic criteria for mood and anxiety disorders and the following references: 2, 9, 11, 20, 21, $23,42-46$. 
Burnout is more common than generally believed. The frequency of high scores in at least one of the three dimensions of the MBI was $70.14 \%$ [12]. The selected participants were 67 on-call physicians at ICUs in Maceió, Brazil. Burnout was diagnosed when high scores were observed in at least one of the three dimensions. The frequency of high scores in all three dimensions of the MBI together was $17.91 \%$, while the frequencies of a high score in each of the three dimensions analyzed separately were $41.79 \%$ for emotional exhaustion, $37.31 \%$ for depersonalization, and $58.2 \%$ for inefficiency.

\section{Managing burnout}

Although the treatment and prevention of occupational burnout primarily lie in the implementation of proper organizational changes, developing proper coping skills is an essential element of prevention and treatment. Choosing the most effective approach depends on our knowledge of burnout types and their dynamics.

Determining the type of burnout is important since it conditions the type of treatment. Farber [13] proposed three types of burnout: a "worn-out" subtype, a classic (frenetic) subtype, and an under-challenged subtype. In the case of a "worn-out" subtype, a person essentially "gives up". When confronted with too much stress or too little gratification, a person with a worn-out subtype becomes apathetic and lacks enthusiasm. In the "classic" burnout, a person works to the point of exhaustion. However, it is itself insufficient for burnout to occur. In the "under challenged" subtype of burnout, a person is negatively affected by monotonous work. Farber criticized viewing burnout as a single phenomenon and stated that different types of burnout may require different forms of treatment [14]. Meanwhile, Montero-Marín et al. [15] made an attempt to define and systematize the properties that characterize Farber's typology proposal. The classification criterion for this systematized typology is based on the level of dedication at work: high in "frenetic" subjects (active coping style), intermediate in "under-challenged" workers and low in "worn-out" subjects (passive coping style). The "frenetic" burnout subtype is characterized by the investment of a large amount of time in work and is common in highly-involved, ambitious and overloaded individuals. The "under-challenged" burnout subtype is influenced by the occupation type. It appears in indifferent and bored individuals who do not find a chance for personal

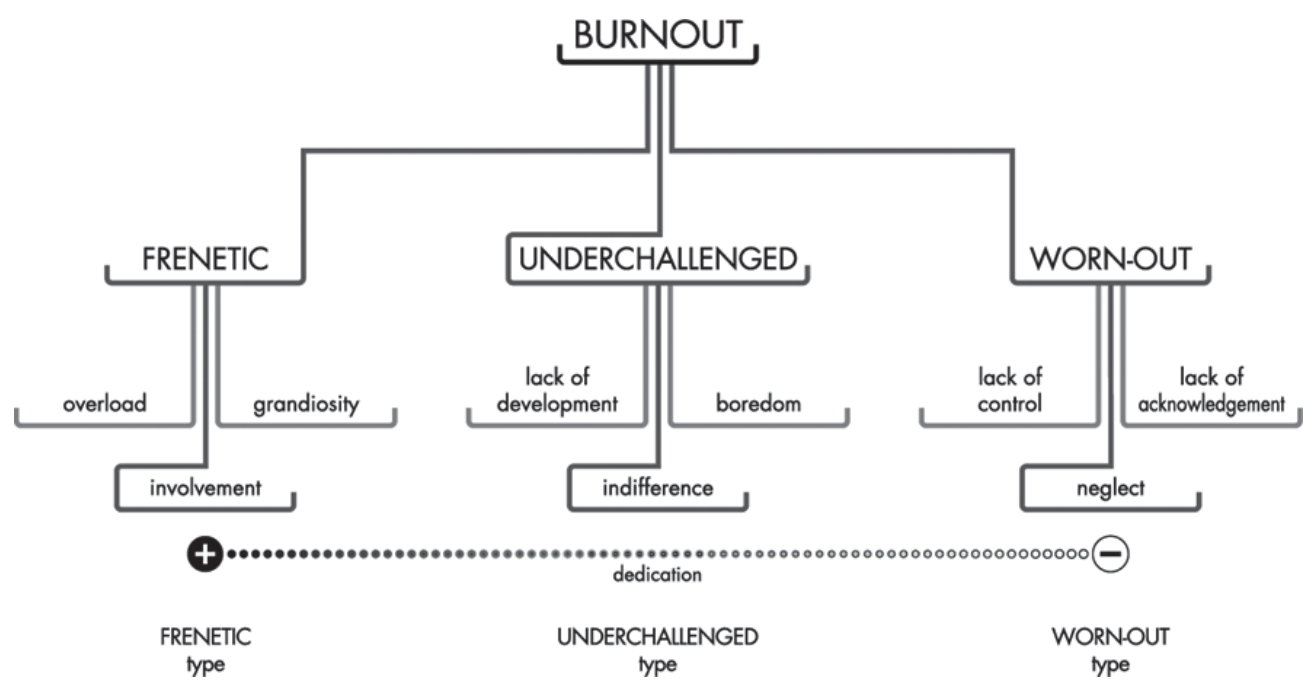

Based on references: 15, 52 (with permission of J. Montero-Marín).

Fig. 1. Structure of systematized typology of under-challenged 
development in their work. The "worn-out" burnout subtype is determined by the rigidity of the organizational structure of an individual's workplace and is characterized by the lack of control over results, lack of recognition for efforts and neglecting responsibilities [16]. This is illustrated by Figure 1.

According to Farber [14], "Psychoanalytic insight, cognitive restructuring, empathic concern, and stress-reduction techniques may all be necessary, albeit in different combinations, to successfully treat the burnout of each type". The "worn-out" teachers manifested symptoms similar to depression, including a perceived loss of self-esteem [14]. According to Farber [14], this type of burnout often requires cognitive approaches that aim to rebalance his or her perceptions. By contrast, a classically burned-out teacher is often helped by a more psychodynamic approach that focuses on the person's strong need for great achievement. In the "under-challenged" type of burnout, teachers could benefit from assertiveness training [14]. Further research is needed to determine the most effective therapy approach in different types of burnout.

In time, family physicians ought to be aware of such classification and refer the patient to a psychiatrist, who should be able to classify burnout correctly: this will be the second most important medical decision to be made. We recommend the use of the "Burnout Clinical Subtype Questionnaire" (BCSQ), in its extended version or the BCSQ-36, or its short version, or the BCSQ-12, introduced by MonteroMarín. It is the first clinical burnout scale introduced by psychiatrists that makes it possible to establish the burnout type. Empirical evidence was provided for the content validity, and relationships with relevant sociodemographic and laboral variables were described $[15,16]$.

The classical Maslach description of burnout does not include the frenetic profile. The Burnout Clinical Subtype Questionnaire is quite a useful tool for the evaluation and choosing specific treatment strategies based on the type of burnout.

\section{Medical treatment of burnout}

Medical treatment of burnout is mostly symptomatic: it involves measures to prevent and treat the symptoms. Since symptoms vary in their degree of severity from person to person, there is an individual approach in developing an intervention treatment plan that targets the occurring symptoms as well as the accompanying disorders such as anxiety, restlessness, headaches, sleep disturbances, problems associated with concentrating, constant feeling of fatigue, irritability, major depression, increased sensitivity to pain, susceptibility to infections, muscle tension, dizziness, gastrointestinal disorders, hypertension, cardiovascular problems and other disorders.

Symptoms of stress can be relieved temporarily with drugs, although they do not remove the cause of stress. The therapeutic benefit of anxiolytics and sedatives is well-established. Anxiolytics and sedatives induce sedation by reducing irritability and psychomotor agitation. Benzodiazepines and barbiturates potentiate gamma-aminobutyric acid (GABA), which produces calming effects and is the most important inhibitory neurotransmitter in the central nervous system (CNS).

Anxiolytics are prescribed when the expected benefits of the treatment outweigh the potential hazards. The major concern is the risk of developing an addiction. People who have difficulty dealing with stress, anxiety or sleep disorders may overuse or become dependent on sedatives. Benzodiazepines and other anxiolytics, even at therapeutic doses, can lead to dependence after only a brief period of use [17]. There are also serious paradoxical reactions that may occur in conjunction with the use of sedatives that result in unexpected outcomes in some individuals. Lader and Morton [18] estimated the incidents of these adverse reactions to be about $5 \%$, even in case of short-term use. The paradoxical reactions may consist of depression, with or without suicidal tendencies, phobias, violent behavior and symptoms sometimes misdiagnosed as psychosis [19]. Anxiolytics sometimes cause memory loss (i.e. amnesia). 
Lorazepam is one such pharmacological agent that can cause anterograde amnesia. Physicians need to consider the long-term side effects of any psychotropic drug. Memory impairment itself could lead in future to decreased work performance and therefore is a risk factor for burnout.

Burnout is characterized by impaired sleep. Stress disrupts the circadian rhythmic secretion of cortisol. The circadian rhythm of hormones and mental performance were investigated in male cadets during a five-day military training course with continuous heavy physical activities and with almost total lack of food and sleep [20]. The 24-h means for androstenedione, dihydroepiandrosterone (DHEA), 17 alpha-hydroxyprogesterone, testosterone and thyroid-stimulating hormone decreased strongly during the course, and the circadian rhythm was reduced to below the minimum levels measured during the control tests. Mental performance also decreased. It is therefore suggested that impaired sleep may play a role in the development of fatigue in burnout [21].

When insomnia interferes with daytime functioning, a short-term treatment with hypnotics is recommended. Hypnotics ought to be prescribed in the lowest effective dosage for a short period of time. If there is a need for a long term treatment, intermittent therapy should be recommended [22]. Prolonged use is discouraged because tolerance can develop. The optimal treatment is to combine hypnotics with Cognitive Behavior Therapy (CBT) [22]. Cognitive-behavioral strategies are more difficult to implement and take longer, but the effects are longer lasting, up to two years after the treatment has ended. These strategies include sleep hygiene, education, relaxation training, stimulus control, and cognitive therapy [22].

Low doses of some antidepressants at bedtime may improve sleep, e.g. doxepin 25 to $50 \mathrm{mg}$, paroxetine 5 to $20 \mathrm{mg}$, trazodone $50 \mathrm{mg}$, trimipramine 75 to $200 \mathrm{mg}$ [22]. However, antidepressants should be used in these low doses mainly when standard hypnotics are not tolerated (rare) or in higher doses when depression is present [22]. Therefore, antidepressants are considered as the last resort in treating insomnia in burnout after the administration of standard hypnotics.

\section{Controversies}

The use of antidepressants for treating burnout remains controversial. Often, antidepressants are prescribed by family doctors without taking into account patients' specific physiological profiles. One of the reasons of prescribing antidepressants to burnout patients is their sedative effect. According to a study conducted at the Finnish Institute of Occupational Health in Helsinki, Finland, antidepressants comprised the most commonly used drug group, especially among employees with severe burnout [23]. This study used data from a nationally representative Finnish sample of 3276 employees, aged from 30 to 64 years. Researchers in this study pointed out that the use of antidepressant medication did not seem to be fully explained by the presence of a depressive or anxiety disorder among the employees with severe burnout. Those with severe burnout were given antidepressants, anxiolytics, and hypnotics more often than those without burnout.

In this study, burnout was measured using the Maslach Burnout Inventory-General Survey (MBI-GS) [11]. The items were scored on a seven-point frequency rating scale ranging from 0 (never) to 6 (daily). High scores for exhaustion and cynicism and low scores for professional efficacy are indicative of burnout. The items of professional efficacy were reversed (lack of professional efficacy). To assess the level of burnout, researchers calculated the weighted total score of the dimensional scores so that exhaustion, cynicism, and lack of professional efficacy had different weights in the syndrome $(0.4 \times$ exhaustion $+0.3 \times$ cynicism $+0.3 \times$ lack of professional efficacy). Burnout and the dimensional scores were categorized as follows: no burnout (scores: 0-1.49), mild burnout (scores: 1.50-3.49), and severe burnout 
Table 2. Laboratory findings in burnout, mood and anxiety disorders

\begin{tabular}{lccccc}
\hline \multicolumn{1}{c}{ Laboratory test } & $\begin{array}{c}\text { Major } \\
\text { depressive } \\
\text { disorder }\end{array}$ & $\begin{array}{c}\text { Generalized } \\
\text { anxiety disorder }\end{array}$ & $\begin{array}{c}\text { Post-traumatic } \\
\text { stress disorder }\end{array}$ & $\begin{array}{c}\text { Acute stress } \\
\text { disorder }\end{array}$ & Burnout \\
\hline Morning plasma cortisol & $\uparrow$ & $\uparrow$ & $\downarrow$ & $\uparrow$ & $\downarrow$ \\
Adrenocorticotropic hormone (ACTH) & $\uparrow$ & & $\mathrm{n}$ or $\downarrow$ & & $\mathrm{n}$ or $\downarrow$ \\
Dehydroepiandrosterone sulphate (DHEA-S) & $\downarrow$ & & & \\
\hline $\mathrm{n}$ - normal levels. & & & & \\
$\uparrow$ - increased levels. & & & & \\
$\downarrow$ - decreased levels. & & & \\
Based on references: $7,8,24,25,42,46-50$. & & & & \\
\end{tabular}

Table 3. A low-dose dexamethasone suppression test in burnout vs. mood and anxiety disorders

\begin{tabular}{ll}
\hline \multicolumn{1}{c}{ Condition/disorder } & \multicolumn{1}{c}{$\begin{array}{l}\text { Dexamethasone } \\
\text { suppression test }\end{array}$} \\
\hline Burnout & hypersuppression of cortisol \\
Major depressive disorder & no suppression of cortisol \\
Posttraumatic stress disorder & hypersuppression of cortisol \\
\hline
\end{tabular}

Based on references: 42, 47, 49, 51.

(scores: 3.50-6). According to this categorization, in severe burnout, the symptoms were experienced approximately daily or weekly, in mild burnout, they occurred monthly, and in the case of no burnout, they were experienced only a few times a year or never [23].

Another important finding in this study was that employees with burnout were less often the targets of occupational interventions than those without burnout, despite the emphasis on work conditions in the etiology of burnout. These findings demonstrate that the current situation in the prevention and treatment of burnout is not optimal.

Although burnout and depression have similar symptoms, there are substantial differences on the physiological level. It was shown that emotional stress can lead to an elevated plasma level of norepinephrine, cortisol and free fatty acids [24]. During acute stress, the plasma cortisol levels rise, but during chronic stress, cortisol levels decline, leading to the state of depletion.
Burnout symptoms are significantly related to endocrine functioning. Severe burnout symptoms are associated with a lower level or smaller increase of the cortisol awakening response (CAR), higher dehydroepiandrosterone-sulphate (DHEAS) levels, lower cortisol/DHEAS ratios and stronger suppression as measured by the dexamethasone suppression test (DST). Such results support the view that burnout symptoms are associated with HPA-axis functioning (Tables 2 and 3).

Antidepressants directly increase the expression and function of corticosteroid receptors in the brain, thus enhance the negative-feedback and reduce the HPA axis activity. They also directly increase the intracellular concentrations of cortisol, but not of corticosterone [26,27]. In addition to this, they directly inhibit membrane steroid transporters that expel glucocorticoids out of the cells. By blocking these transporters, antidepressants increase the intracellular levels of glucocorticoids. The findings also support the role of steroid transporters in the effects of antidepressants on GR function [26,27]. The authors demonstrated that coincubation of antidepressants and glucocorticoids leads to increased GR function if the glucocorticoids are a substrate for the transporters (dexamethasone and cortisol). Decreased GR function was also observed when glucocorticoid was not a substrate for the transporters (corticosterone). It is essential to know the mechanisms of action of antidepressants and 
their potential effects on patients with depression and burnout.

According to the new research at the Center for Studies on Human Stress at the Lafontaine Hospital in Montreal [24], antidepressants, having the effect of lowering the cortisol levels, could reduce these levels in burnout patients even more and potentially put them at a higher risk of spiralling into burnout. The researches opt for the development of evidence-based approaches and technologies that distinguish burnout from depression. The same researchers suggest that it would be important for physicians and psychiatrists to do a series of cortisol tests. Really high or really low cortisol levels are warnings of potential burnout and also the risk of mental health issues in general. The saliva cortisol test could help employees realize they need to amend their lives to reduce their stress, and also help doctors steer the patients at risk into stress reduction programs. The researchers mentioned above also suggest that testing saliva for cortisol could be useful in predicting burnout and it could help people with symptoms of burnout avoid being put on medication that might actually make the condition worse. Research about the possible relationships between the subtypes of burnout and psychophysiological variables, such as the levels of cortisol, could be quite helpful in choosing the most appropriate treatment for each particular subtype.

\section{Developing new treatment approaches}

The influence of cortisol on brain functioning and its role in glucocorticoid feedback is commonly accepted. The penetration of cortisol in the human brain is less extensive than that of corticosterone [28]. The ratio of corticosterone over cortisol in the brain was significantly increased comparably to plasma. The research suggests that corticosterone has a more prominent role in human brain functioning than had previously been recognized. The preferential uptake of corticosterone in the human brain over cortisol in stress disorders could lead toward development of novel selective steroids for the treatment of stress-related disorders.

Some clinicians use very low doses of cortisol (1.25-2.5 mg/ day) in patients with a low plasma cortisol level [29]. Research indicates that the best course of action is first to evaluate and treat hormonal deficiencies with hormones prior to prescribing psychotropic medications [30]. Some serotonin reuptake inhibitors (SSRIs) such as paroxetine and sertraline could increase cortisol levels in some depressed patients [31].

An in-patient study was conducted to compare the effects of $10 \mathrm{mg}$ and $30 \mathrm{mg}$ doses of intravenous naloxone and nalmefene in healthy volunteers [32]. Significant increases in ACTH and cortisol levels were observed after both antagonists, without an apparent dose-response relationship. Almefene injection resulted in greater HPA axis activation than either dose of naloxone. Researchers suggest that kappa- and delta-opioids may play important roles in the regulation of the HPA axis. Nalmefene may be useful as a pharmacotherapeutic agent for HPA-axis regulation. Opioid antagonists activate the HPA-axis. They may have a desirable effect in severe burnout patients when the HPAaxis is hypofunctioning. In this study, increased HPA-axis activity was observed in healthy volunteers. It is hard to predict whether opioid antagonists will be as effective in burnout patients as in healthy individuals. Future studies are needed to determine the effects of opioid antagonists in burnout. On the other hand, the activation of opioid receptors decreases HPA-axis activity. Endogenous opioid peptides (POMC, beta-endorphin, enkephalins and dynorphins) and the endocannabinoid system (ECS; anandamide, 2-arachidonoylglycerol) also regulate the HPA-axis. Considering the current knowledge on possible pathomechanisms of the burnout syndrome, the activation of opioid and cannabinoid receptors is promising in burnout prevention and treatment since it improves the mood and enhances the ability to cope with stress. The endocannabinoid system (eCB) has been found to control the neurotransmitter release from several 
neuron populations (e.g. GABA, glutamate, catecholamines and monoamines), suggesting a general mechanism for tuning neuronal activity, and thereby regulating the responses to emotion and stress.

Activating the eCB has been suggested by targeting endocannabinoid-degrading enzymes to prolong the precisely regulated pro-homeostatic action of endocannabinoids [33]. Regulation of the endocannabinoid levels presents an opportunity to examine its effects in burnout prevention and treatment.

\section{Dietary supplementation}

When addressing burnout in patient rehabilitation, a state of depletion needs to be taken into account. Vitamins and minerals are crucial in addressing adrenal and HPA axis dysfunction. Cortisol synthesis is dependent on adequate supplies of various vitamins and minerals. The serum levels of calcium, potassium, zinc, iron and copper are reduced under cortisol secretion. Vitamin C, Niacin derivatives, pantothenic acid and folic acid are important to maintain steroid secretion from the adrenal cortex [34].

Supplementation with omega-3 fatty acids inhibits the adrenal activation elicited by mental stress [35]. After three weeks of fish oil supplementation, the cortisol spike following a stress test was abolished and the epinephrine spike was significantly blunted [36]. The norepinephrine concentration was significantly decreased in the experimental group with docosahexaenoic acid (DHA) intake, whereas its levels did not change in the control group [37]. Research suggests that these effects of DHA intake may be applied to people under psychological stress.

Krähenbühl et al. [38] examined the inhibitory effects of 18 beta-Glycyrrhetinic acid (GRA) on 11 beta-hydroxysteroid dehydrogenase (11 beta OHSD), the enzyme responsible for conversion of cortisol to inactive cortisone. GRA is a major metabolite of glycyrrhizic acid (glycyrrhizin), an important constituent of licorice and licorice root. In vivo, increasing doses of GRA were associated with reduced cortisone concentration and increased the cortisol/cortisone ratio. Daily doses of $1.5 \mathrm{~g}$ can constantly inhibit 11 beta OHSD.

Another study [39] proposed that an acceptable daily intake of glycyrrhizin is $0.015-0.229 \mathrm{mg}$ glycyrrhizin $/ \mathrm{kg}$ body weight per day, based on in vivo and clinical evidence. The researchers report that licorice root extracts can be used to maintain cortisol levels, when taken in smaller doses, and that severe hypocortisolism may require three or four separate doses throughout the day.

A number of supplements have traditionally been used as sedatives, such as L-theanine, Passiflora incarnata, Valeriana officinalis, Humulus lupulus, Matricaria chamomilla, Galphimia glauca, Bacopa monniera, Centella asiatica, Melissa officinalis, Piper methysticum, Scutellaria lateriflora, and Ziziphus jujuba [40].

Numerous botanicals have been listed as "adaptogens". An adaptogenic herb is defined as a substance that increases the body's ability to resist stress and exerts a balancing effect on various systems of the body such as the immune, central nervous and cardiovascular systems. Adaptogens under this definition include Eleutherococcus senticosus (Siberian Ginseng), Panax Ginseng, Rhodiola rosea, Schisandra chinensis, Withania somnifera (Ashwaghanda), Astragalus membranaceus, Scutellaria baicalensis and others. Panossian and Wikman [41] suggest that "adaptogenic herbs work by inducing specific heat-shock proteins within cells, which protects both cells and organs during stress-induced changes in homeostasis". Further research studies are required to determine the efficacy of the supplements in humans and especially in burnout patients.

\section{CONCLUSION}

Evidently, today's medicine has not yet developed sufficient methods for treating medical burnout, although it is possible to categorize the burnout problem according to previous studies. There is no doubt that acute and chronic stress affects human health and the mortality rate. 
However, this issue is not addressed to a great degree by conventional Medicine. Chronic stress-related disorders often fall outside the category of a "true" disease and are frequently treated as depression or not treated at all. It is necessary to identify a source of chronic stress and develop strategies to cope with it by problem solving or by implementing organizational changes. The goal is knowing how to perceive and respond to a stressor in ways that do not stimulate the HPA-axis.

In severe burnout, taking a leave from work may not be enough for rehabilitation. Medical intervention should address not only mood changes, but physiological changes as well. More and more literature works suggest that the evaluation of the HPA axis should be brought to the attention of primary care physicians. There is no universal agreement on specific treatment and diagnostic measures to evaluate the wide range of HPA axis disorders. The cost-effective evaluation of adrenal hormones via saliva samples by a primary care physician may significantly alter the course of therapy in numerous chronic disease patients. Developing burnout screening techniques for family physicians, implementing proper diet, as well as nutritional and lifestyle changes for supporting the HPA function are likely to benefit patients.

Hopefully, DSM-V may recognize burnout as a medical disorder. This would help in differentiating burnout from similar clinical syndromes such as depression or anxiety. Psychiatric disorders may have similar symptoms, but they have distinctive hormonal profiles. Having burnout recognized as a distinct medical condition would help to provide appropriate treatment to patients suffering from it. Proper treatment is essential for a fast and full recovery. Besides health benefits, shortening the recovery time will have a huge impact on the global economy by reducing the number of sick-leave days and increasing the general wellbeing of workers. There is a need for both further research and development of evidence-based good practices to recognize and treat occupational burnout.

\section{REFERENCES}

1. Appels A. Why do imminent victims of a cardiac event feel so tired? Int J Clin Pract 1997;51:447-50.

2. Maslach C, Schaufeli WB, Leiter MP. Job burnout. Annu Rev Psychol 2001;52:397-422.

3. Hallman T, Thomsson H, Burell G, Lisspers J, Setterlind S. Stress, burnout and coping: differences between women with coronary heart disease and healthy matched women. J Health Psychol 2003;8(4):433-45.

4. Appels A, Mulder P. Fatigue and heart disease. The association between 'vital exhaustion' and past, present and future coronary heart disease. J Psychosom Res 1989;33:727-38.

5. Pedersen SS, Middel B. Increased vital exhaustion among type-D patients with ischemic heart disease. J Psychosom Res 2001;51(2):443-9.

6. Räikkönen K, Lassila R, Keltikangas-Järvinen L, Hautanen A. Association of chronic stress with plasminogen activator inhibitor-1 in healthy middle-aged men. Arterioscler Thromb Vasc Biol 1996;16(3):363-67.

7. Keltikangas-Järvinen L, Räikkönen K, Hautanen A, Adlercreutz H. Vital exhaustion, anger expression, and pituitary and adrenocortical hormones. Implications for the insulin resistance syndrome. Arterioscler Thromb Vasc Biol 1996;16(2): 275-80.

8. Nicolson NA, van Diest R. Salivary cortisol patterns in vital exhaustion. J Psychosom Res 2000;49(5):335-42.

9. Sandstrom A, Rhodin IN, Lundberg M, Olsson T, Nyberg L. Impaired cognitive performance in patients with chronic burnout syndrome. Biol Psychol 2005;69:271-9.

10. Esterling BA, Kiecolt-Glaser JK, Bodnar JC, Glaser R. Chronic stress, social support, and persistent alterations in the natural killer cell response to cytokines in older adults. Health Psychol 1994;13:291-8.

11. Maslach C, Jackson SE, Leiter MP. Maslach Burnout Inventory. Palo Alto, CA: Consulting Psychologist Press; 1996.

12. Barbosa FT, Leao BA, Tavares GM, Santos JG. Burnout syndrome and weekly workload of on-call physicians: Crosssectional study. Sao Paulo Med J 2012;130(5):282-8. 
13. Farber BA. Burnout in psychotherapists: Incidence, types, and trends. Psychother Priv Pract 1990;8(1):35-44.

14. Farber BA. Treatment strategies for different types of teacher burnout. J Clin Psychol 2000;56:675-89.

15. Montero-Marín J, García-Campayo J, Mosquera Mera D, López del Hoyo Y.A new definition of burnout syndrome based on Farber's proposal. J Occup Med Toxicol 2009;4(31):1-17.

16. Montero-Marín J, García-Campayo J, Fajó-Pascual M, Carrasco JM, Gascón S, Gili M, et al. Sociodemographic and occupational risk factors associated with the development of different burnout types: The cross-sectional University of Zaragoza study. BMC Psychiatry 2011;11(49):1-13.

17. Trickett S. Withdrawal from benzodiazepines. J R Coll Gen Pract 1983;33:608.

18. Lader M, Morton S. Benzodiazepine problems. Br J Addict 1991;86:823-8.

19. Michelini S, Cassano GB, Frare F, Perugi G. Long-term use of benzodiazepines: Tolerance, dependence and clinical problems in anxiety and mood disorders. Pharmacopsychiatry 1996;29:127-34.

20. Opstad K. Circadian rhythm of hormones is extinguished during prolonged physical stress, sleep and energy deficiency in young men. Eur J Endocrinol 1994;131:56-66.

21. Ekstedt M, Söderström M, Akerstedt T, Nilsson J, Søndergaard HP, Aleksander P. Disturbed sleep and fatigue in occupational burnout. Scand J Work Environ Health 2006;32(2):121-31.

22. Doghramji K. Approach to the patient with a sleep or wakefulness disorder. The Merck Manual Online. [cited 2013 June 20]. Available from http:/www.merckmanuals.com/professional/neurologic_disorders/sleep_and_wakefulness_disorders/approach_ to_the_patient_with_a_sleep_or_wakefulness_disorder.html

23. Ahola K, Honkonen T, Virtanen M, Kivimäki M, Isometsä E, Aromaa A, et al. Interventions in relation to occupational burnout: The population-based health 2000 study. J Occup Environ Med 2007;49(9):943-52.

24. Juster RP, Sindi S, Marin MF, Perna A, Hashemi A, Pruessner JC, et al. A clinical allostatic load index is associated with burnout symptoms and hypocortisolemic profiles in healthy workers. Psychoneuroendocrinology 2011;36(6):797-805.

25. Sonnenschein M, Mommersteeg PM, Houtveen JH, Sorbi MJ, Schaufeli WB, van Doornen LJ. Exhaustion and endocrine functioning in clinical burnout: An in-depth study using the experience sampling method. Biol Psychol 2007;75(2): 176-84.

26. Pariante CM, Hye A, Williamson R, Makoff A, Lovestone S, Kerwin RW. The antidepressant clomipramine regulates cortisol intracellular concentrations and glucocorticoid receptor expression in fibroblasts and rat primary neurones. Neuropsychopharmacology 2003;28:1553-61. DOI: 10.1038/ sj.npp.1300195.

27. Pariante CM, Kim RB, Makoff A, Kerwin RW. Antidepressant fluoxetine enhances glucocorticoid receptor function in vitro by modulating membrane steroid transporters. Br J Pharmacol 2003;139:1111-8.

28. Karssen AM, Meijer OC, van der Sandt I, Lucassen PJ, de Lange EC, de Boer AG, et al. Multidrug resistance P-glycoprotein hampers the access of cortisol but not of corticosterone to mouse and human brain. Endocrinology 2001;142(2): 686-94.

29. Hotze SF. Adrenal Fatigue \& Cortisol [cited 2013 June 20]. Available from http://www.cushings-help.com/downloads/ adrenal.pdf

30. Schuder SE. Stress-Induced Hypocortisolemia Diagnosed as Psychiatric Disorders Responsive to Hydrocortisone Replacement. Ann N Y Acad Sci 2005;1057:466-78.

31. Sagud M, Pivac N, Mück-Seler D, Jakovljević M, MihaljevićPeles A, Korsić M. Effects of sertraline treatment on plasma cortisol, prolactin and thyroid hormones in female depressed patients. Neuropsychobiology 2002;45:139-43.

32. Schluger JH, Ho A, Borg L, Porter M, Maniar S, Gunduz $\mathrm{M}$, et al. Nalmefene causes greater hypothalamic-pituitaryadrenal axis activation than naloxone in normal volunteers: Implications for the treatment of alcoholism. Alcohol Clin Exp Res 1998;22(7):1430-36.

33. Petrosino S, Di Marzo V. FAAH and MAGL inhibitors: Therapeutic opportunities from regulating endocannabinoid levels. Curr Opin Invest Drugs 2010;11(1):51-62. 
34. Guilliams TG, Edwards L. Chronic stress and the HPA axis: Clinical assessment and therapeutic considerations. The review of natural \& neutraceutical therapies for clinical practice. Standard 2010;9(2):1-12.

35. Hamazaki K, Itomura M, Huan M. Effect of omega-3 fatty acid-containing phospholipids on blood catecholamine concentrations in healthy volunteers: A randomized, placebo-controlled, double-blind trial. Nutrition 2005;21: $705-10$

36. Delarue J, Matzinger O, Binnert C. Fish oil prevents the adrenal activation elicited by mental stress in healthy men. Diabetes Metab 2003;29:289-95.

37. Hamazaki T, Itomura M, Sawazaki S, Nagao Y. Antistress effects of DHA. Biofactors 2000;13:41-5.

38. Krähenbühl S, Hasler F, Frey BM, Frey FJ, Brenneisen R, Krapf R. Kinetics and dynamics of orally administered 18 beta-glycyrrhetinic acid in humans. J Clin Endocrinol Metab 1994;78(3):581-5.

39. Isbrucker RA, Burdock GA. Risk and safety assessment on the consumption of Licorice root (Glycyrrhiza sp.), its extract and powder as a food ingredient, with emphasis on the pharmacology and toxicology of glycyrrhizin. Regul Toxicol Pharmacol 2006;46(3):167-92.

40. Head KH, Kelly GS. Nutrients and botanicals for treatment of stress: Adrenal fatigue, neurotransmitter imbalance, anxiety, and restless sleep. Altern Med Rev 2009;14(2):114-40.

41. Panossian A, Wikman G. Evidence-based efficacy of adaptogens in fatigue, and molecular mechanisms related to their stress-protective activity. Curr Clin Pharmacol 2009;4(3): 198-219.

42. Pruessner JC, Hellhammer DH, Kirschbaum C. Burnout, perceived stress, and cortisol responses to awakening. Psychosom Med 1999;61(2):197-204.
43. Clarke DM, Kissane DW. Demoralization: Its phenomenology and importance. Aust N Z J Psychiatry 2002;36(6):733-42.

44. Gabel S. Demoralization in mental health organizations: Leadership and social support help. Psychiatr Q 2012;83(4): 489-96.

45. West CP, Dyrbye LN, Satele DV, Sloan JA, Shanafelt TD. Concurrent validity of single-item measures of emotional exhaustion and depersonalization in burnout assessment. Gen Intern Med 2012;27(11):1445-52.

46. Greaves-Lord K, Ferdinand RF, Oldehinkel AJ, Sondeijker FE, Ormel J, Verhulst FC. Higher cortisol awakening response in young adolescents with persistent anxiety problems. Acta Psychiatr Scand 2007;116(2):137-44.

47. Kanter ED, Wilkinson CW, Radant AD, Petrie EC, Dobie DJ, McFall ME, et al. Glucocorticoid feedback sensitivity and adrenocortical responsiveness in posttraumatic stress disorder. Biol Psychiatry 2001;50(4):238-45.

48. Moch SL, Panz VR, Joffe BI, Havlik I, Moch JD. Longitudinal changes in pituitaryadrenal hormones in South African women with burnout. Endocrine 2003;21(3):267-72.

49. Kellner M, Yehuda R, Arlt J, Wiedemann K. Longitudinal course of salivary cortisol in post-traumatic stress disorder. Acta Psychiatr Scand 2002;105(2):153-5

50. Yehuda R, Brand SR, Golier JA, Yang RK. Clinical correlates of DHEA associated with post-traumatic stress disorder. Acta Psychiatr Scand 2006;114(3):187-93.

51. Tiller JW, Biddle N, Maguire KP, Davies BM. The dexamethasone suppression testand plasma dexamethasone ingeneralized anxiety disorder. Biol Psychiatry 1988;23(3):261-70.

52. Montero-Marín J. The 'Burnout Clinical Subtype Questionnaire' (BCSQ-36/BCSQ-12): A new definition of burnout through a differential characterization of the syndrome. $\mathrm{Hu}$ esca: Ediciones GH; 2012. p. 106.

This work is available in Open Access model and licensed under a Creative Commons Attribution-NonCommercial 3.0 Poland License - http://creativecommons.org/ licenses/by-nc/3.0/pl/deed.en. 